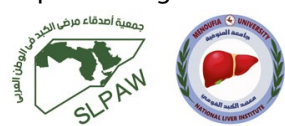

\title{
Efficacy and safety of sofosbuvir and daclatasvir in patients with chronic hepatitis $C$ virus induced cirrhosis with Child- Pugh class B
}

Mayssa El Raziky ${ }^{1,2}$, Sherif Hamdy ${ }^{1,2}$, Yasser Hamada ${ }^{1}$, Nermin Mohamed Abdelaziz ${ }^{3}$, Mohamed Hassany ${ }^{4}$, Wahid Doss ${ }^{1}$ and Zeinab Zakaria ${ }^{1 *}$ (D)

\begin{abstract}
Background: This study aimed to evaluate the efficacy, safety and tolerability of SOF/DCV \pm RBV in a cohort of Egyptian patients with chronic hepatitis C (CHC)-induced cirrhosis with decompensation (class B7-B9).

Results: After excluding the 9 patients who withdrew, SVR12 rate according to per protocol analysis was $82.9 \%$ (92/111), non-response and relapse rates were $2.7 \%$ (3/111) for each, 4 patients died secondary to hematemesis, and $8.1 \%$ stopped therapy due to worsening of Child's class. SVR12 rate was significantly higher among patients with higher baseline WBCs count and lower among patients with Child-Pugh class B9. All treatment intolerant patients had ascites in pre-treatment assessment $(P=0.02)$. There was a significant decline in the levels of hemoglobin, ALT and AST, and serum bilirubin $(P<0.001)$ and a significant increase in albumin level $(P<0.001)$ at the end of treatment when compared to their pre-treatment levels. Follow-up of the three HCC did not show evidence of tumor recurrence.
\end{abstract}

Conclusions: The SOF/DCV combination \pm ribavirin is an effective and safe regimen for patients with $\mathrm{CHC}$ induced cirrhosis with mild decompensation. Treatment did not increase the risk of HCC recurrence.

Keywords: Chronic hepatitis C-induced cirrhosis, Mild decompensation, SOF/DCV, SVR12

\section{Background}

Egypt had the highest prevalence of hepatitis $\mathrm{C}$ virus infection in the world, and genotype 4 virus is found in more than $90 \%$ of patients [1]. Chronic hepatitis C $(\mathrm{CHC})$ has been linked to rise in mortality, utilization of resources, and deterioration of patients' well-being [2].

The initial goal of hepatitis $C$ treatment is to eradicate hepatitis $\mathrm{C}$ virus $(\mathrm{HCV})$ which is specified by a sustained virological response (SVR), defined as undetectable $\mathrm{HCV}$

\footnotetext{
*Correspondence: zenab.zakaria@yahoo.com

1 Department of Endemic Medicine and Hepato-Gastroenterology,

Faculty of Medicine, Cairo University, Cairo, Egypt

Full list of author information is available at the end of the article
}

RNA after end of treatment for 12 weeks (SVR 12) or 24 weeks (SVR 24) [3].

Since 2014 several direct acting antivirals (DAAs) have been approved enabling interferon-free antiviral treatments with high SVR rates, and some of them are giving the chance for decompensated (cirrhotic) patients [4].

Sofosbuvir (SOF) is an inhibitor of NS5B-polymerase.It has effectiveness against all genotypes with a high resistance barrier and good tolerability with once daily oral dosing [5]. Daclatasvir (DCV) is an inhibitor of NS5Apolymerase and has a high antiviral activity [6]. Reported results from previous clinical studies demonstrated that SOF and DCV combination therapy, have a good tolerability, limited drug to drug interactions, and a high rates 
of SVR [7]. Interestingly, this treatment regimen is effective in patients who are difficult to cure [8], despite that, liver cirrhosis is the most important negative predictor of SVR to DAAs therapies [3].

The current study aimed to evaluate the efficacy, safety, and tolerability of SOF/DCV \pm RBV in a cohort of Egyptian patients with $\mathrm{CHC}$ induced decompensated cirrhosis with Child scoreB7-B9.

\section{Methods}

This is a prospective observational study that was initiated on February 2016 at Cairo Fatemic Hospital (one of the assigned specialized centers for treatment of viral hepatitis in Egypt). Target population included 120 patients with $\mathrm{HCV}$-induced liver cirrhosis with mild decompensation (Child-Pugh class B7-B9) and all treated with sofosbuvir and daclatasvir \pm RBV. HCV genotyping was not tested prior to therapy as $90 \%$ of Egyptians are HCV4 [9] and on real-life scenario testing was not proven cost effectiveness.

Ethical committee approval was obtained from National Committee for Control of Viral Hepatitis (NCCVH) before enrollment in the study [10].

Patients had cirrhosis as evidenced by transabdominal ultrasonography, or transient elastography showing liver stiffness of $>12.5 \mathrm{Kpa}$ and had evidence of mild decompensation (Child-Pugh class B7-B9).

Patients with history of HCC were included 24 weeks after intervention aiming at cure with no evidence of activity by dynamic imaging (CT or MRI). If serum creatinine ranged from 1.5 to $2.5 \mathrm{mg} / \mathrm{dl}$, eGFR should be exceeding $30 \mathrm{ml} / \mathrm{min}$ with favorable nephrological consultation.

Patients with platelet count $<50,000 / \mathrm{mm}^{3}$, serum creatinine $>2.5 \mathrm{mg} / \mathrm{dl}$, pregnancy, or inability to use effective contraception, co-infection with HBV or HIV, history of clinically significant illness or any other major medical disorder that may interfere with treatment, and chronic liver disease of non-HCV etiology were excluded from the study.

All patients provided written informed consent before enrolling to the study.

\section{Treatment}

Patients were treated according to EASL recommendations on treatment of hepatitis C 2015 (EASL 2015). Nine patients withdrew from therapy (all were treatment naïve) and 9 patients stopped treatment due to adverse events thus 102 patients completed treatment most of them $(73 / 102 ; 71.6 \%)$ received treatment in the form of sofosbuvir $(400 \mathrm{mg})$, daclatasvir $(60 \mathrm{mg})$, and daily weight-based ribavirin for 12 weeks; in these patients, ribavirin was started at the dose of $600 \mathrm{mg}$ daily and the dose subsequently adjusted depending on tolerance every 2 weeks aiming at reaching $1000 \mathrm{mg}$. Twenty nine patients $(28.4 \%)$ received treatment in the form of SOF (400 mg) and DCV (60 mg) for 24 weeks; the cause of this treatment extension was related to RBV intolerance.

Potential drug-drug interactions with patients' medications were checked using the university of Liverpool application on smart phones (liver-pool HEP i chart) drugs contraindicated with SOF or DAC were discontinued or changed. No dose adjustment for SOF or DAC was done (www.hep-druginteractions.org).

\section{Pre-treatment assessment}

Age, gender, previous treatment status (naïve or experienced), and if experienced (details about previously administered medications), Child-Pugh score and MELD score were recorded.

Serological tests (anti HCV antibody, HBsAg), quantitative PCR for HCV RNA, ALT, and AST, C.B.C (hemoglobin level, W.B.Cs count, and platelet count), serum albumin level, serum bilirubin level, I.N.R, serum creatinine, and AFP were tested.

Ultrasound (US) to detect the progression of fibrosis with characteristic changes such as a coarse or nodular appearance of the parenchyma, hepatomegaly, and caudate lobe hypertrophy, evidence of portal hypertension (portal vein diameter, velocity of flow, flow reversal), presence of focal lesions, ascites, and splenomegaly [11].

All routine parameters were tested at baseline, at the end of treatment and at week 12 after the end of treatment. These include blood count, AST, ALT, serum bilirubin, serum albumin, INR, serum creatinine, and Alfa fetoprotein and abdominal ultrasonography. Whereas CBC, AST, ALT, serum albumin, serum bilirubin, and creatinine were additionally tested while on treatment (W4, W8).

\section{Efficacy assessment}

HCV RNA level determination was done at screening (baseline), at the end of treatment (week 12 or 24) and at 12 weeks after the end of treatment. HCV RNA was measured using the ROCHE COABA TaqMan HCV version 2.0 (lower limit of detection $15 \mathrm{IU} / \mathrm{ml}$ ). Primary virological outcome was achieved at SVR12 defined as undetectable HCV RNA at 12 weeks following the completion of treatment. Virological failure was defined as non-response (detectable HCV RNA levels at the end of treatment period) or relapse (detectable HCV RNA level during follow-up in a patient with an undetectable level at the end of treatment period). 


\section{Safety assessment}

Safety endpoints included graded adverse events, serious adverse events, discontinuation due to adverse events, deaths, and laboratory abnormalities. Adverse events were considered serious if they were causing deterioration, resulted in discontinuation of HCV therapy, required hospitalization, resulted in death, or considered severe by the treating physician.

\section{Statistical analysis}

Data were fed to the computer and analyzed using IBM SPSS software package version 20.0. Quantitative data were described using median (minimum and maximum) for non-parametric variables and mean \pm standard deviation for parametric variables after testing normality using Kolmogrov-Smirnov test. Significance of the obtained results was judged at the 5\% level. One-way ANOVA was used for parametric quantitative variables, to compare between more than two studied groups. For non-parametric quantitative variables, Kruskal-Wallis test was used to compare between more than two studied groups with Mann-Whitney $U$ test to study within group significance. Paired $t$ and Wilcoxon signed rank tests were used to compare two periods (before and after treatment) for parametric and non-parametric variables, respectively. All significant factors in univariate analysis were further analyzed in multivariate analysis.

\section{Results}

Patients' ages ranged from 36 to 70 years with a mean of 55.83 years \pm 7.9 . There was a female predominance among the studied patients (60.8\%).

Most of the studied patients were treatment naïve $(97 / 120 ; 80.8 \%)$ while $(23 / 120 ; 19.2 \%)$ received prior IFN based therapy or sofosbuvir based therapy).

Regarding laboratory parameters, median platelet count was $92.5 \times 10^{3} / \mathrm{ml}$, median $\mathrm{HCV}$ viral load was $326 \times 10^{3} \mathrm{IU} / \mathrm{ml}$, mean serum albumin was $2.85 \mathrm{gm} /$ $\mathrm{dl}$, median serum bilirubin was $1.37 \mathrm{mg} / \mathrm{dl}$, and mean serum creatinine was $0.84 \mathrm{mg} / \mathrm{dl}$. Regarding MELD score, the mean value was $11.3,33$ patients $(27.5 \%)$ had a score of $<10$, and 87 patients $(72.5 \%)$ had a score of $\geq$ 10. Most patients were among class B7 (80/120; 66.7\%) followed by class B8 (31/120;25.8\%) and only 9 patients (9/120; 7.5\%) were class B9.

Transabdominal ultrasonography showed that 60 patients $(50 \%)$ had mild ascites. Focal hepatic lesions (FHL) were detected in 2 patients one of them had hemangioma and the other had hepatocellular carcinoma (HCC) well ablated before starting treatment with no evidence of activity by triphasic C.T.
Nine patients were missed at follow-up (all were treatment naïve) and 9 patients stopped treatment because of intolerance thus 102 patients completed treatment most of them $(73 / 102 ; 71.6 \%)$ received treatment in the form of SOF/DCV and RBV for 12 weeks and 29 patients $(28.4 \%)$ received treatment in the form of SOF/DCV (when RBV was not tolerated) for 24 weeks.

A sustained virological response rate 12 (SVR12) of $82.9 \%$ was achieved, $81.1 \%$ in treatment naïve and $87 \%$ in treatment experienced. SVR 12 rates were $75 \%, 80.6 \%$, and $77.7 \%$ in $\mathrm{B} 7, \mathrm{~B} 8$, and $\mathrm{B} 9$ respectively.

Three patients (2.7\%) showed positive PCR at the end of treatment and were considered non-responders, 3 patients $(2.7 \%)$ showed negative PCR at the end of treatment and positive PCR at week 12 post-therapy and were considered relapsers,

Among the non-responders, two were treatment naïve (66.7\%) and one was treatment experienced (33.3\%). All of them $(100 \%)$ were of class B7. The relapsers were treatment naïve. One in each of class B7, B8, and B9.

Concerning safety and tolerability: treatment discontinuation was higher among patients who received (SOF $+\mathrm{DCV}+\mathrm{RBV}$ ) regimen. It was higher among patients of Child-Pugh class B8, most discontinuation occurred at week 8 . Causes of discontinuation were worsening of jaundice (serum bilirubin above $5 \mathrm{mg} / \mathrm{dl}$ ) in 3 patients, development of severe anemia not responding to reducing the dose of RBV (hemoglobin below $7.5 \mathrm{gm} / \mathrm{dl}$ ) in 2 patients, worsening hepatic decompensation in 4 patients (2 patients developed hepatic encephalopathy and 2 patients developed massive ascites). Additionally 4 deaths were reported due to uncontrolled hematemesis, all reported deaths were among patients who received $(\mathrm{SOF}+\mathrm{DCV}+\mathrm{RBV})$ regimen, all of them (100\%) developed at week 12 .

Univariate and multivariate analysis of predictors of SVR12 in treated patients showed that: WBCs count was significantly higher among patients who achieved SVR12. Other laboratory parameters did not have a significant effect on SVR12. Patients with Child-Pugh class B9 failed to achieve SVR12 (Table 1).

Baseline characteristics of patients who failed to achieve SVR12 are shown in (Table 2).

Comparison between laboratory results at baseline and at the end of treatment among the studied patients revealed a significant decline in hemoglobin concentration $(P=0.001)$, ALT levels $(P=0.001)$, AST levels $(P=$ $0.001)$, serum bilirubin level $(P=0.019)$, and AFP $(P=$ $0.001)$, as well as a significant increase in serum albumin level $(P=0.001)$ (Table 3$)$.

Laboratory results at baseline and at the end of treatment in relation to treatment outcome showed that 
Table 1 Univariate and multivariate analysis of predictors of SVR12 in treated patients

\begin{tabular}{|c|c|c|c|c|c|c|}
\hline \multirow[t]{2}{*}{ Parameter } & \multirow{2}{*}{$\begin{array}{l}\text { Patients who achieved SVR12 } \\
N=92\end{array}$} & \multirow{2}{*}{$\begin{array}{l}\text { Patients } \\
\text { who failed } \\
\text { treatment } \\
N=15\end{array}$} & \multicolumn{2}{|c|}{ Univariate analysis } & \multicolumn{2}{|c|}{ Multivariate analysis } \\
\hline & & & $P$ value & $\mathrm{RR} \% \mathrm{Cl})$ & $P$ value & $\begin{array}{l}\text { RR } \\
(95 \% \mathrm{Cl})\end{array}$ \\
\hline \multicolumn{7}{|l|}{ Age (years) } \\
\hline Mean \pm SD & $55.05 \pm 8.13$ & $57.8 \pm 7.29$ & 0.22 & 1.0(0.97-1.13) & & \\
\hline (Min-Max) & $(36-69)$ & $(41-70)$ & & & & \\
\hline \multicolumn{7}{|l|}{ Gender } \\
\hline Male & $31(33.7 \%)$ & $9(60 \%)$ & 0.06 & $2.9(0.96-9.05)$ & & \\
\hline Female (r) & $61(66.3 \%)$ & $6(40 \%)$ & & 1 & & \\
\hline \multicolumn{7}{|l|}{ Prior HCV treatment } \\
\hline Naïve & $72(78.3 \%)$ & $12(80 \%)$ & 0.88 & $0.9(0.23-3.5)$ & & \\
\hline Experienced $(r)$ & $20(21.7 \%)$ & $3(20 \%)$ & & 1 & & \\
\hline \multicolumn{7}{|l|}{ Hemoglobin (gm/dl) } \\
\hline Male: (13.2-17.5 gm/dl) & $12.17 \pm 1.6$ & $12.02 \pm 1.57$ & 0.75 & $0.9(0.67-1.33)$ & & \\
\hline Female: (11.5-16 gm/dl) & & & & 1 & & \\
\hline $\mathrm{WBCs} * 10^{3} / \mathrm{m}\left(411 \times 10^{3} / \mathrm{ml}\right)$ & $4.66 \pm 1.6$ & $3.75 \pm 1.49$ & 0.05 & $\begin{array}{l}0.66(0.44-1) \\
1\end{array}$ & 0.04 & $0.64(0.42-0.98)$ \\
\hline $\begin{array}{l}\text { Platelet count } \times 10^{3} / \mathrm{ml}\left(150-450 \times 10^{3} /\right. \\
\mathrm{ml})\end{array}$ & $94.5(50-356)$ & $84(55-142)$ & 0.16 & $0.99(0.97-1.004)$ & & \\
\hline $\mathrm{PCR}(\mathrm{iu} / \mathrm{ml})^{*} 10^{3}$ & $297.10(0.135-20223)$ & $437(9.97-4823)$ & 0.36 & $1.0(1.0-1.0)$ & & \\
\hline ALT/ULN (fold) & $1.325(0.22-5.35)$ & $0.98(0.48-4.12)$ & 0.35 & $0.66(0.27-1.58)$ & & \\
\hline AST/ULN (fold) & $1.765(0.4-5.85)$ & $1.73(0.4-4.7)$ & 0.94 & $0.98(0.59-1.64)$ & & \\
\hline Serum Albumin (g/dl) $(3.4-5.4 \mathrm{~g} / \mathrm{dl})$ & $2.8 \pm 0.4$ & $2.79 \pm 0.26$ & 0.31 & $0.45(0.09-2.09)$ & & \\
\hline Serum bilirubin (mg/dl) (0.3-1 mg/dl) & $1.42 \pm 0.7$ & $1.74 \pm 0.87$ & 0.15 & $1.68(0.84-3.39)$ & & \\
\hline I.N.R. (0.9-1.2) & $1.32 \pm 0.22$ & $1.34 \pm 0.18$ & 0.60 & $1.93(0.1623 .2)$ & & \\
\hline \multicolumn{7}{|l|}{ Serum creatinine (mg/dl) } \\
\hline Male: (0.6-1.2 mg/dl) & $0.85 \pm 0.25$ & $0.99 \pm 0.41$ & 0.07 & $4.6(0.88-23.8)$ & & \\
\hline \multicolumn{7}{|l|}{ Female: (0.8-1.4 mg/dl) } \\
\hline $\operatorname{AFP}(\mu \mathrm{g} / \mathrm{l})(<8 \mu \mathrm{g} / \mathrm{l})$ & $12.35(0.1-201)$ & $9(2.64-83)$ & 0.4 & $0.98(0.96-1.02)$ & & \\
\hline \multicolumn{7}{|l|}{ MELD score } \\
\hline Mean \pm SD & $11 \pm 2.97$ & $12.87 \pm 2.6$ & & & & \\
\hline (Min-max) & $(6-17)$ & $(8-18)$ & & & & \\
\hline$<10(r)$ & $29(31.5 \%)$ & $2(13.3 \%)$ & 0.15 & 1 & & \\
\hline$\geq 10$ & $63(68.5 \%)$ & 13(86.7\%) & & $2.9(0.63-14.1)$ & & \\
\hline \multicolumn{7}{|l|}{ Ultrasonography } \\
\hline Liver cirrhosis & $92(100 \%)$ & $15(100 \%)$ & 1 & Undefined & & \\
\hline Splenomegaly & 55 (59.8\%) & $6(40 \%)$ & 0.2 & $0.45(0.15-1.4)$ & & \\
\hline Ascites & $44(47.8 \%)$ & $11(73.3 \%)$ & 0.07 & $3.0(0.89-10.1)$ & & \\
\hline Hemangioma & $0(0 \%)$ & $1(6.67 \%)$ & 0.15 & U’ndefined & & \\
\hline Ablated HCC & $1(1.09 \%)$ & $0(0 \%)$ & 1.0 & Undefined & & \\
\hline \multicolumn{7}{|l|}{ Child-Pugh score } \\
\hline B7 & 67 (72.8\%) & $6(40 \%)$ & 0.18 & 1 & & 1 \\
\hline B8 & $20(21.7 \%)$ & $6(40 \%)$ & 0.06 & $3.4(0.97-11.5)$ & 0.07 & $3.2(0.9-11.6)$ \\
\hline B9 & $5(5.4 \%)$ & $3(20 \%)$ & 0.024 & $6.7(1.3-35.1)$ & 0.016 & $8.6(1.5-49.8)$ \\
\hline
\end{tabular}

baseline WBCs was significantly lower $(P=0.02)$, and baseline serum creatinine was significantly higher among patients who discontinued therapy due to adverse events(intolerants) $(p=0.003)$ than other outcome groups. As regards mean hemoglobin level, median platelet count, median ALT/ULN, median AST/ULN, mean serum albumin level, median serum bilirubin level, mean I.N.R, and median AFP level, there was no statistically significant difference among 
Table 2 Baseline characteristics of patients who failed to achieved SVR12

\begin{tabular}{|c|c|c|c|c|}
\hline Parameter & $\begin{array}{l}\text { Non-responders } \\
N=3\end{array}$ & $\begin{array}{l}\text { Relapsers } \\
N=3\end{array}$ & $\begin{array}{l}\text { Discontinued due to } \\
\text { adverse events } \\
N=9\end{array}$ & $P$ value \\
\hline \multicolumn{5}{|l|}{ Age (years) } \\
\hline Mean \pm SD & $56.33 \pm 6.66$ & $51.33 \pm 8.13$ & $60.44 \pm 6.24$ & 0.18 \\
\hline$($ Min-Max) & $(49-62)$ & $(41-61)$ & $(52-70)$ & \\
\hline \multicolumn{5}{|l|}{ Gender } \\
\hline Male & $1(33.3 \%)$ & $2(66.7 \%)$ & $6(66.7 \%)$ & 0.57 \\
\hline Female & $2(66.7 \%)$ & $1(33.3 \%)$ & $3(33.3 \%)$ & \\
\hline \multicolumn{5}{|l|}{ Prior HCV treatment } \\
\hline Naïve & $2(66.7 \%)$ & $3(100 \%)$ & $7(77.8 \%)$ & 0.57 \\
\hline Experienced & 1(33.3\%) & $0(0 \%)$ & $2(22.2 \%)$ & \\
\hline \multicolumn{5}{|l|}{ Laboratory data } \\
\hline Hemoglobin (gm/dl) & $13.3 \pm 2.3$ & $12.73 \pm 1.11$ & $11.36 \pm 1.32$ & 0.14 \\
\hline \multicolumn{5}{|l|}{ Male: $(13.2-17.5 \mathrm{gm} / \mathrm{dl})$} \\
\hline \multicolumn{5}{|l|}{ Female: (11.5-16 gm/dl) } \\
\hline $\mathrm{WBCs} \times 10^{3} / \mathrm{ml}\left(4-11 \times 10^{3} / \mathrm{ml}\right)$ & $3.3 \pm 0.36$ & $5.7 \pm 2.1$ & $3.26 \pm 1.12$ & 0.035 \\
\hline Platelet count $\times 10^{3} / \mathrm{ml}\left(150-450 \times 10^{3} / \mathrm{ml}\right)$ & $58(58-100)$ & $84(74-111)$ & $111(55-142)$ & 0.33 \\
\hline $\mathrm{PCR}(\mathrm{IU} / \mathrm{ml}) \times 10^{3}$ & $4780(216.218-4823)$ & $1210(393-1490)$ & $429(10.780-1603.390)$ & 0.19 \\
\hline ALT/ULN (fold) & $1.05(0.78-4.12)$ & $1.03(0.8-1.24)$ & $0.93(0.48-2.85)$ & 0.49 \\
\hline AST/ULN (fold) & $1.62(1.03-4.7)$ & $1.85(1.38-3.41)$ & $1.73(0.4-3.46)$ & 0.91 \\
\hline Serum albumin $(\mathrm{g} / \mathrm{dl})(3.4-5.4 \mathrm{~g} / \mathrm{dl})$ & $3 \pm 0.26$ & $2.53 \pm 0.25$ & $2.8 \pm 0.24$ & 0.10 \\
\hline Serum bilirubin $(\mathrm{mg} / \mathrm{dl})(0.3-1 \mathrm{mg} / \mathrm{dl})$ & $1.77 \pm 0.6$ & $2.3 \pm 0.79$ & $1.54 \pm 1$ & 0.48 \\
\hline I.N.R. (0.9-1.2) & $1.42 \pm 0.28$ & $1.42 \pm 0.14$ & $1.3 \pm 0.18$ & 0.53 \\
\hline \multicolumn{5}{|l|}{ Serum creatinine (mg/dl) } \\
\hline Male: $(0.6-1.2 \mathrm{mg} / \mathrm{dl})$ & $0.6 \pm 0.1$ & $0.87 \pm 0.4$ & $1.17 \pm 0.4$ & 0.09 \\
\hline \multicolumn{5}{|l|}{ Female: (0.8-1.4 mg/dl) } \\
\hline $\operatorname{AFP}(\mu \mathrm{g} / \mathrm{l})(<8 \mu \mathrm{g} / \mathrm{l})$ & $12(6.5-83)$ & $10.2(9-20.7)$ & $7.6(2.64-25)$ & 0.37 \\
\hline \multicolumn{5}{|l|}{ MELD score } \\
\hline Median & 12 & 14 & 13 & \\
\hline$($ Min-max) & $(11-13)$ & $(11-17)$ & $(8-18)$ & 0.62 \\
\hline$<10$ & $0(0 \%)$ & $0(0 \%)$ & $2(22.2 \%)$ & 0.46 \\
\hline$\geq 10$ & $3(100 \%)$ & $3(100 \%)$ & $7(77.8 \%)$ & \\
\hline \multicolumn{5}{|l|}{ Ultrasonographic features } \\
\hline Liver cirrhosis & $3(100 \%)$ & $3(100 \%)$ & $9(100)$ & $P=1.0$ \\
\hline Splenomegaly & 1(33.3\%) & $1(33.3 \%)$ & $4(44.4 \%)$ & $P=0.9$ \\
\hline Ascites & 1(33.3\%) & $1(33.3 \%)$ & $9(100 \%)$ & $P=0.02$ \\
\hline Hemangioma & 1(33.3\%) & $0(0 \%)$ & $0(0 \%)$ & $P=0.12$ \\
\hline Ablated HCC & $0(0 \%)$ & $0(0 \%)$ & $0(0 \%)$ & \\
\hline \multicolumn{5}{|l|}{ Child-Pugh score } \\
\hline B7 & $3(100 \%)$ & $1(33.3 \%)$ & $2(22.2 \%)$ & 0.19 \\
\hline B8 & $0(0 \%)$ & $1(33.3 \%)$ & $5(55.6 \%)$ & \\
\hline B9 & $0(0 \%)$ & $1(33.3 \%)$ & $2(22.2 \%)$ & \\
\hline
\end{tabular}

different outcome groups neither at baseline nor at the end of treatment (Table 4).

\section{Follow-up}

Trans-abdominal ultrasonography was done for 65 patients at SVR12. The patient with previously ablated
HCC didn't develop any new FHLs, the patient responded to treatment, AFP level was $6.2 \mathrm{IU} / \mathrm{l}$ before starting treatment, raised to $200 \mathrm{IU} / \mathrm{l}$ at W4 then declined to $24 \mathrm{IU} / \mathrm{l}$ at SVR12. New FHLs were detected in 2 patients (3\%). They were females, 49 and 48 years old respectively, first received treatment for 12 weeks and second for 24 weeks, 
Table 3 Comparison between laboratory results at baseline and at the end of treatment among the studied patients

\begin{tabular}{|c|c|c|c|}
\hline & At baseline & At the end of treatment & $P$ value \\
\hline Hemoglobin (gm/dl) & $12.22 \pm 1.6$ & $11.25 \pm 1.5$ & $P<0.001$ \\
\hline WBCs $\times 10^{3} / \mathrm{ml}$ & $4.63 \pm 1.5$ & $4.63 \pm 1.7$ & $P=1.0$ \\
\hline Platelet count $\times 10^{3} / \mathrm{ml}$ & $92.5(50.0-356)$ & $105(37.0-392)$ & $P=0.26$ \\
\hline ALT/ULN (folds) & $1.255(0.22-5.35)$ & $0.67(0.2-2.0)$ & $P<0.001$ \\
\hline AST/ULN (folds) & $1.75(0.4-5.85)$ & $0.9(0.25-7.0)$ & $P<0.001$ \\
\hline Serum albumin (gm/dl) & $2.85 \pm 0.4$ & $3.23 \pm 0.5$ & $P<0.001$ \\
\hline Serum bilirubin (mg/dl) & $1.37(0.3-3.2)$ & $1.2(0.2-3.4)$ & $P=0.019$ \\
\hline I.N.R. & $1.33 \pm 0.24$ & $1.38 \pm 0.24$ & $P=0.16$ \\
\hline Serum creatinine $(\mathrm{mg} / \mathrm{dl})$ & $0.84 \pm 0.2$ & $0.89 \pm 0.3$ & $P=0.08$ \\
\hline AFP (IU/I) & $10.8(0.1-201)$ & $5.8(0.2-24)$ & $P<0.001$ \\
\hline
\end{tabular}

All parameters described as mean \pm SD except platelet count, ALT/ULN, AST/ULN, serum bilirubin, and AFP as median (min-max)

Table 4 Laboratory results at baseline and at the end of treatment in relation to treatment outcome

\begin{tabular}{|c|c|c|c|c|c|c|}
\hline \multicolumn{2}{|l|}{ Outcome } & \multirow{2}{*}{$\begin{array}{l}\text { Non-responders } \\
n=3\end{array}$} & \multirow{2}{*}{$\begin{array}{l}\text { Relapsers } \\
n=3\end{array}$} & \multirow{2}{*}{$\begin{array}{l}\text { Responders } \\
n=92\end{array}$} & \multirow{2}{*}{$\begin{array}{l}\text { Intolerant } \\
n=9\end{array}$} & \multirow[t]{2}{*}{$P$ value } \\
\hline Laboratory result & & & & & & \\
\hline \multirow[t]{2}{*}{ Hemoglobin (HGB) concentration (gm/dl) } & Baseline & $13.3 \pm 2.3$ & $12.73 \pm 1.11$ & $12.17 \pm 1.6$ & $11.36 \pm 1.32$ & $P=0.26$ \\
\hline & End of treatment & $12.07 \pm 0.31$ & $12.13 \pm 0.38$ & $11.2 \pm 1.5$ & & $P=0.36$ \\
\hline \multirow[t]{2}{*}{ White blood cell count (WBCs) $\left({ }^{*} 10^{3} / \mu l\right)$} & Baseline & $3.3 \pm 0.36$ & $5.7 \pm 2.1^{\mathrm{A}}$ & $4.66 \pm 1.6^{\mathrm{B}}$ & $3.26 \pm 1.12^{\mathrm{AB}}$ & $P=0.02$ \\
\hline & End of treatment & $3.67 \pm 0.23$ & $5.0 \pm 1.8$ & $4.65 \pm 1.7$ & & $P=0.58$ \\
\hline \multirow[t]{2}{*}{ platelet count (PLTs) $\left(* 10^{3} / \mu \mathrm{l}\right)$} & Baseline & $58(58-100)$ & $84(74-111)$ & $94.5(50-356)$ & $111(55-142)$ & $P=0.39$ \\
\hline & End of treatment & $57(43-138)$ & $105(100-170)$ & $104(37-392)$ & & $P=0.32$ \\
\hline \multirow[t]{2}{*}{ Serum creatinine (mg/dl) } & Baseline & $0.6 \pm 0.1^{A}$ & $0.87 \pm 0.4$ & $0.85 \pm 0.25^{B}$ & $1.17 \pm 0.4^{\mathrm{AB}}$ & $P=0.003$ \\
\hline & End of treatment & $0.78 \pm 0.09$ & $0.85 \pm 0.07$ & $0.89 \pm 0.37$ & & $P=0.86$ \\
\hline \multirow[t]{2}{*}{ ALT/ULN (folds) } & Baseline & $1.05(0.78-4.12)$ & $1.03(0.8-1.24)$ & $1.325(0.22-5.35)$ & $0.93(0.48-2.85)$ & $P=0.13$ \\
\hline & End of treatment & $0.4(0.4-1.29)$ & $1.0(0.88-1.05)$ & $0.67(0.2-2)$ & & $P=0.13$ \\
\hline \multirow[t]{2}{*}{ AST/ULN (folds) } & Baseline & $1.62(1.03-4.7)$ & $1.85(1.38-3.41)$ & $1.765(0.46-5.85)$ & $1.73(0.4-3.46)$ & $P=0.9$ \\
\hline & End of treatment & $0.55(0.48-2.27)$ & $0.86(0.6-1.43)$ & $0.9(0.25-7)$ & & $P=0.77$ \\
\hline \multirow[t]{2}{*}{ Albumin level (gm/dl) } & Baseline & $3.0 \pm 0.26$ & $2.53 \pm 0.25$ & $2.89 \pm 0.4$ & $2.8 \pm 0.24$ & $P=0.32$ \\
\hline & End of treatment & $3.5 \pm 0.2$ & $3.1 \pm 0.14$ & $3.23 \pm 0.48$ & & $P=0.87$ \\
\hline \multirow[t]{2}{*}{ Serum bilirubin (mg/dl) } & Baseline & $1.77 \pm 0.6$ & $2.3 \pm 0.79$ & $1.42 \pm 0.7$ & $1.54 \pm 1.0$ & $P=0.23$ \\
\hline & End of treatment & $1.35 \pm 0.07$ & $2.13 \pm 1.07$ & $1.29 \pm 0.7$ & & $P=0.17$ \\
\hline \multirow[t]{2}{*}{ I.N.R. } & Baseline & $1.42 \pm 0.28$ & $1.42 \pm 0.14$ & $1.32 \pm 0.22$ & $1.3 \pm 0.18$ & $P=0.72$ \\
\hline & End of treatment & $1.39 \pm 0.15$ & $1.32 \pm 0.0$ & $1.38 \pm 0.25$ & & $P=0.97$ \\
\hline \multirow[t]{2}{*}{$\operatorname{AFP}(\mu \mathrm{g} / \mathrm{l})$} & Baseline & $12.0(6.5-83)$ & $10.2(9.0-20.7)$ & $12.35(0.1-201)$ & $7.6(2.64-25)$ & $P=0.51$ \\
\hline & End of treatment & $4.8(4.8-4.8)$ & $8.15(6.7-9.6)$ & $5.7(0.2-24)$ & & $P=0.49$ \\
\hline
\end{tabular}

both were treatment naïve and Child-Pugh score B7. The focal lesions were hyperechoic, in segment IV and measured $6 \mathrm{~mm}$ and $12 \mathrm{~mm}$ in diameter respectively, AFP level was $22 \mathrm{IU} / \mathrm{l}$ at baseline and $6.3 \mathrm{IU} / \mathrm{l}$ at SVR12 in first one and was $17 \mathrm{IU} / \mathrm{l}$ at baseline and $4.2 \mathrm{IU} / \mathrm{l}$ at SVR12 in second one. Triphasic CT examination confirmed these findings.

One patient developed rising serum creatinine (at baseline serum creatinine was $2 \mathrm{mg} / \mathrm{dl}$. eGFR $=32 \mathrm{ml} / \mathrm{min}$ with favorable nephrological consultation, at SVR12 serum creatinine, rose to $3.3 \mathrm{mg} / \mathrm{dl}$ the patient started hemodialysis.
She was a 62 years old diabetic and hypertensive female, with Child-Pugh B7, treatment experienced (previously received sofosbuvir and ribavirin). She was re-treated for 24 weeks; however, ribavirin was stopped at week 12 due to development of anemia (hemoglobin $=8 \mathrm{gm} / \mathrm{dl}$ ). She showed aSVR.

\section{Discussion}

This study reports safety and efficacy of SOF/DCV combination regimen for treating patients with chronic hepatitis $\mathrm{C}$-induced cirrhosis with Child-Pugh class 
B7-B9. An overall SVR12 rate of $82.9 \%$ (81.8\% among treatment naïve and $87 \%$ among treatment experienced patients) is reported; this unexpected higher response rate among treatment experienced patients may be due to small number of patients in this subset; nonresponse rate was $2.7 \%$, and relapse rate was $2.7 \%$ while $8.1 \%$ of patients discontinued therapy due to adverse events. Patients with decompensated cirrhosis (ChildPugh $B / C$ ) have lower response rates than patients with compensated cirrhosis (Child-Pugh A). Reasons for these lower response rates may include reduced drug delivery due to shunting leading to $\mathrm{HCV}$ reservoirs, altered drug metabolism and uptake due to impaired liver synthetic function, or impaired immune responses which are present in cirrhotic patients. Relatively few patients with decompensated liver disease have been enrolled in DAA trials or real-life studies [12-17].

In the present study, the mean age of patients was 55.83 years \pm 7.9. Similarly, in Maan et al.s study the mean age was 57.1 years, and in Fernández Carrillo et al.'s study the mean age was 55 years $[15,16]$. While in Calvaruso et al.s study the mean age was 65.4 years [17].

Regarding gender distribution: our study showed female predominance $(60.8 \%)$, in contrast to Maan et al. 2016, Fernández Carrillo et al. 2017, and Calvaruso et al. 2018 who showed male predominance (62.3\%), (67\%), and $(56.9 \%)$ respectively [14-16]. Our patients were recruited from governmentally supported program (nonhealth insurance) who is concerned with treating unemployed persons mostly housewives.

In the currently discussed study, $80.8 \%$ of patients were treatment naïve while only $19.2 \%$ were treatment experienced. This was similar to El-khayat et al's study where $78.4 \%$ of patients were treatment naïve and $21.6 \%$ were treatment experienced [13]. While in Maan et al's study $41.2 \%$ of patients were treatment naïve and $58.8 \%$ were treatment experienced among CTP class B/C patients [16], and in Fernández Carrillo et al.s study treatment experienced patients represent $57 \%$ of CTP class B/C patients [15].

Baseline mean serum albumin level was $2.85 \mathrm{gm} / \mathrm{dl}$, median serum bilirubin level was $1.37 \mathrm{mg} / \mathrm{dl}$, and median platelet count was $92.5 \times 10^{3} / \mathrm{ml}$; these were different from other studies where Fernández Carrillo et al's study showed a median serum albumin level of $3.2 \mathrm{gm} / \mathrm{dl}$, a median serum bilirubin level of $2.1 \mathrm{mg} / \mathrm{dl}$, and a median platelet count of $62 \times 10^{3} / \mathrm{ml}$ [15], and in Mann et al.s study, the baseline mean serum albumin level for $\mathrm{CP}$ class $\mathrm{B} / \mathrm{C}$ patients was $3.1 \mathrm{gm} / \mathrm{dl}$ and the median platelet count was $75 \times 10^{3} / \mathrm{ml}[16]$.

Baseline trans-abdominal ultrasonography revealed that $50 \%$ of patients had mild ascites and that 2 patients had FHLs one of them $(0.83 \%)$ was hemagnioma and the other was an ablated HCC as confirmed by triphasic C.T. This was different from Omar et al's study who reported that ascites was present in only $0.1 \%$ of patients who received SOF/DAC and $0.3 \%$ of patients who received SOF/DAC/RBV and that ablated FHLs was present in $0.1 \%$ of patients who received SOF/DAC and in $0.6 \%$ of patients who received SOF/DAC/RBV [12].

Patients in our study were more tolerant to ribavirin, $71.6 \%$ of them received treatment for 12 weeks this was higher than that reported by Fernández Carrillo et al. 2017 who stated that $46 \%$ of patients received treatment for 12 weeks [15]. In other studies, Omar et al. treatednaïve patients without cirrhosis without RBV, and those who had cirrhosis or were treatment-experienced (interferon experienced or SOF experienced) received RBV, and El-Khayat et al. reported a multicenter study of patients with liver cirrhosis genotype 4; in his study all patients received SOF $(400 \mathrm{mg})$ and DCV $(60 \mathrm{mg})$ daily in addition to weight-based ribavirin (RBV) for 12 weeks and when RBV is contraindicated the treatment duration was extended to 24 weeks $[12,13]$.

In current study, 3 patients (2.7\%) relapsed. This was similar to that reported by El-khayat et al. who reported an overall relapse rate of $2 \%$, and was lower than that reported by Fernández Carrillo et al. who concluded that relapse rate among $\mathrm{CP}$ class $\mathrm{B} / \mathrm{C}$ patients was $14 \%$ $[13,15]$. The ETR was higher among treatment naïve and treatment experienced patients than that reported by El-Khayat et al's study after excluding Child's class A patients [13].

In our study, 9 patients (8.1\%) discontinued therapy due to development of adverse events most of them suffered from worsening decompensation (rising serum bilirubin, development of hepatic encephalopathy or development of massive ascites) and some of them suffered from anemia. Similarly, Fernández Carrillo et al. also reported that incident decompensation was the most common serious adverse event and that it occurred in overall 7\% of patients [15]. While Omar et al. found that treatment was prematurely discontinued in only $1.5 \%$ of patients and that the most common events leading to discontinuation were patient withdrawal and pregnancy. They also reported that the most frequent reported adverse events were hematological, decompensation, and/or development of ascites and that serious adverse events were reported in six patients receiving SOF/DCV [12].

In our study, 4 patients (3.6\%) died during follow-up. This was lower than that reported by Fernández Carrillo et al. who reported a death rate of $6.4 \%$ among CTP class B/C patients [14] and was slightly lower than that reported by Maan et al. who reported a death rate of 5/114 (4.4\%) among CP class B/C patients [16]. 
Our analysis of data showed that age, gender, prior $\mathrm{HCV}$ treatment status, did not have a significant effect on SVR12, while baseline WBCs count was significantly higher among patients who achieved SVR12 (may have a better immunological response) and that serum creatinine level was significantly higher among intolerant patients who discontinued therapy due to adverse events, the presence of ascites also increased the risk of intolerance (possibly more advanced liver disease). El-Khayat et al. also reported that age, treatment experience, and viral load did not have a significant effect on SVR but reported that female gender, MELD score $<10$, and platelet count $>150,000 / \mathrm{mm}^{3}$ were significantly associated with higher rate of SVR12 [13]. Omar et al. reported that treatment experience and viral load did not have a significant effect on SVR12 but reported that age, gender, ALT, AST, albumin, bilirubin, WBCs count, hemoglobin, platelet, and INR were significantly different between patients who achieved SVR12 and those who did not [12]. In Maan et al.s study analysis of data among patients with $\mathrm{CP}$ class $\mathrm{B} / \mathrm{C}$ revealed that age, gender, prior treatment status, serum bilirubin level, and platelet count did not increase the risk for hepatic decompensation, but they reported that serum albumin level $<35 \mathrm{~g} / \mathrm{l}$ and MELD score $\geq 14$ increased the risk of hepatic decompensation [16].

To assess the safety during treatment, a comparison between results of laboratory investigations at baseline and at the end of treatment revealed that there was a significant improvement in liver biochemical profile in the form of reduction in the level of AST $(P<0.001)$, ALT $(P$ $<0.001)$, and serum bilirubin $(P=0.019)$, and an increase in the level of serum albumin $(P<0.001)$. There was also a significant decline in the level of hemoglobin $(P<0.001)$ and AFP $(P<0.001)$, this was in agreement with Fernández Carrillo et al. 2017 who reported that treatment was associated with improvements in MELD scores, particularly in CTP class B/C patients [15].

As previously reported and described in the literature, few cases of recurrence of formerly treated $\mathrm{HCC}$ or even de novo lesions after HCV treatment are expected [17]. We repeated the trans-abdominal ultrasonography for 65 patients 12 weeks post-therapy, and revealed that the patient with previously ablated HCC did not develop any new FHLs and that new FHLs developed in $2 / 65$ patients (3\%). Thus, we concluded that treatment did not increase the risk of development of HCC. This was similar to that reported by Waziry et al. and Bang and Song $[18,19]$. But contrasted with that reported by Reig et al. who reported an unexpected increase in the incidence of both de novo (i.e., incident) HCC, recurrent HCC, and more aggressive and faster progression of $\mathrm{HCC}$ in patients treated with DAAs [20]. The 2 patients who developed new FHLs have achieved SVR12 which means that eradication of the virus did not abolish the risk of HCC development. This finding was consistent with that reported by Cardoso et al. 2016 who suggests that we need to consider patients with cirrhosis remain at risk of HCC in spite of eradication of HCV [21].

In the present study, one patient developed rising serum creatinine showing that patients with high baseline serum creatinine may be at risk of worsening renal functions when they receive DAA therapy. This was in agreement with Carrier et al., 2016 who recommended that close monitoring of renal function is required, particularly for at-risk patients (transplanted, HIV-coinfected, CKD, hypertensive, or diabetic patients) [22]. While in another Egyptian study which included patients eligible for treatment with sofosbuvir-based regimens, the reported side effects were fatigue and headache, whereas the liver function tests were significantly improved at SVR12 [23].

\section{Limitations}

No endoscopy was done for patients. Loss of some patients during follow-up. Need for longer follow-up periods.

\section{Conclusion}

The SOF/DCV combination at their standard doses \pm ribavirin is an effective and safe regimen for the management of Egyptian patients with chronic hepatitis C-induced cirrhosis with mild decompensation.

\section{Acknowledgements \\ We would like to thank the NCCVH team members and the members of the executive office.}

\section{Authors' contributions}

This work was carried out in collaboration between all authors. Author MR and SH did study design and chose the research subject. Authors YH, NAA, MH, and WD did analysis, interpretation of the data, and assessment for drug safety and efficacy. Author ZZ did literature searches, drafting of the article, and did critical revision. All authors read and approved the final manuscript.

\section{Funding}

No financial or any other support was supplied.

Availability of data and materials

We have an excel sheet including raw data.

\section{Declarations}

Ethics approval and consent to participate

Ethical committee approval was obtained from National Committee for Control of Viral Hepatitis (NCCVH) before enrollment in the study.

Consent for publication

Not applicable. 


\section{Competing interests}

All authors declare that they have no competing interests.

\section{Author details}

'Department of Endemic Medicine and Hepato-Gastroenterology, Faculty of Medicine, Cairo University, Cairo, Egypt. ${ }^{2}$ Cairo Fatemic Hospital, Cairo, Egypt. ${ }^{3}$ El Sahel Teaching Hospital, Cairo, Egypt. ${ }^{4}$ National Hepatology and Tropical Medicine Research Institute (NHTMRI), Cairo, Egypt.

Received: 2 December 2021 Accepted: 18 January 2022

Published online: 31 January 2022

\section{References}

1. Dore GJ, Ward J, Thursz M (2014) Hepatitis C disease burden and strategies to manage the burden (Guest Editors Mark Thursz, Gregory Dore and John Ward). J Viral Hepat 21(s1):1-4

2. Younossi ZM, Stepanova M, Mishra A, Venkatesan C, Henry L, Hunt S (2013) The impact of chronic hepatitis $C$ on resource utilisation and in-patient mortality for Medicare beneficiaries between 2005 and 2010. Aliment Pharmacol Ther 38(9):1065-1075

3. Zopf S, Kremer AE, Neurath MF, Siebler J (2016) Advances in hepatitis C therapy: What is the current state-what come's next? World J Hepatol 8(3):139

4. American Association for the Study of Liver Diseases \& Infectious Diseases society of America (2014). Recommendation for testing, managing, and treating hepatitis $C$

5. Lawitz E, Mangia A, Wyles D, Rodriguez-Torres M, Hassanein T, Gordon SC et al (2013) Sofosbuvir for previously untreated chronic hepatitis $C$ infection. N Engl J Med 368(20):1878-1887

6. Karino Y, Toyota J, Ikeda K, Suzuki F, Chayama K, Kawakami Y et al (2013) Characterization of virologic escape in hepatitis $C$ virus genotype $1 \mathrm{~b}$ patients treated with the direct-acting antivirals daclatasvir and asunaprevir. J Hepatol 58(4):646-654

7. Wyles DL, Ruane PJ, Sulkowski MS, Dieterich D, Luetkemeyer A, Morgan TR et al (2015) Daclatasvir plus sofosbuvir for HCV in patients coinfected with HIV-1. N Engl J Med 373(8):714-725

8. Estrabaud E, Vidaud M, Marcellin P, Asselah T (2012) Genomics and HCV infection: progression of fibrosis and treatment response. J Hepatol 57:1110-1125

9. Messina JP, Humphreys I, Flaxman A, Brown A, Cooke GS, Pybus OG, Barnes $E$ (2015) Global distribution and prevalence of hepatitis $C$ virus genotypes. Hepatology. 61:77-87

10. El-Akel W, El-Sayed MH, El Kassas M, El-Serafy M, Khairy M, Elsaeed K et al (2017) National treatment programme of hepatitis C in Egypt: Hepatitis C virus model of care. J Viral Hepat 24(4):262-267

11. Sharma S, Khalili K, Nguyen GC (2014) Non-invasive diagnosis of advanced fibrosis and cirrhosis. World J Gastroenterol 20(45):16820

12. Omar H, El Akel W, Elbaz T, El Kassas M, Elsaeed K, El Shazly H et al (2018) Generic daclatasvir plussofosbuvir, with or without ribavirin, in treatment of chronichepatitis C: real-world results from 18378 patients inEgypt. Aliment Pharmacol Ther 47(3):421-431

13. El-Khayat H, Fouad Y, Mohamed HI, El-Amin H, Kamal EM, Maher M, Risk A (2018) Sofosbuvir plus daclatasvir with or without ribavirin in 551 patients with hepatitis C-related cirrhosis, genotype 4. Aliment Pharmacol Ther 47(5):674-679

14. Doss W, Shiha G, Hassany M, Soliman R, Fouad R, Khairy M et al (2015) Sofosbuvir plus ribavirin for treating Egyptian patients with hepatitis $C$ genotype 4. J Hepatol 63(3):581-585

15. Fernández Carrillo C, Lens S, Llop E, Pascasio JM, Crespo J, Arenas J et al (2017) Treatment of hepatitis C virus infection in patients with cirrhosis and predictive value of model for end-stage liver disease: Analysis of data from the Hepa-C registry. Hepatology 65(6):1810-1822

16. Maan R, van Tilborg M, Deterding K, Ramji A, van der Meer AJ, Wong F et al (2016) Safety and effectiveness of direct-acting antiviral agents for treatment of patients with chronic hepatitis $C$ virus infection and cirrhosis. Clin Gastroenterol Hepatol 14(12):1821-1830

17. Calvaruso V, Cabibbo G, Cacciola I, Petta S, Madonia S, Bellia A et al (2018) Incidence of Hepatocellular Carcinoma in Patients with HCV-associated
Cirrhosis Treated with Direct-Acting Antiviral Agents. Gastroenterology 155(2):411-421.e4

18. Waziry R, Hajarizadeh B, Grebely J, Amin J, Law M, Danta M et al (2017) Hepatocellular carcinoma risk following direct-acting antiviral HCV therapy: A systematic review, meta-analyses, and meta-regression. J Hepatol 67(6):1204-1212

19. Bang CS, Song $\| \mathrm{H}$ (2017) Impact of antiviral therapy on hepatocellular carcinoma and mortality in patients with chronic hepatitis C: systematic review and meta-analysis. BMC Gastroenterol 17(1):46

20. Reig M, Boix L, Bruix J (2017) The impact of direct antiviral agents on the development and recurrence of hepatocellular carcinoma. Liver Int 37(S1):136-139

21. Cardoso H, Vale AM, Rodrigues $S$, Gonçalves $R$, Albuquerque A, Pereira $P$, Coelho R (2016) High incidence of hepatocellular carcinoma following successful interferon-free antiviral therapy for hepatitis $C$ associated cirrhosis. J Hepatol 65(5):1070-1071

22. Carrier P, Essig M, Debette-Gratien M, Sautereau D, Rousseau A, Marquet P, Loustaud-Ratti V (2016) Anti-hepatitis C virus drugs and kidney. World J Hepatol 8(32):1343

23. Abd El Rahman MM, Galal GM, Abd El Hamid RM, Abd Allah SK (2020) Efficacy, safety, and biochemical response of sofosbuvir and daclatasvir combination in chronic hepatitis C treated patients in Sohag Governorate. Sohag Med J Effi, Saf Biochem Resp Sofosbuvir 24(1):104-111

\section{Publisher's Note}

Springer Nature remains neutral with regard to jurisdictional claims in published maps and institutional affiliations.

\section{Submit your manuscript to a SpringerOpen ${ }^{\circ}$ journal and benefit from:}

- Convenient online submission

$\checkmark$ Rigorous peer review

- Open access: articles freely available online

- High visibility within the field

- Retaining the copyright to your article

Submit your next manuscript at $\boldsymbol{\nabla}$ springeropen.com 